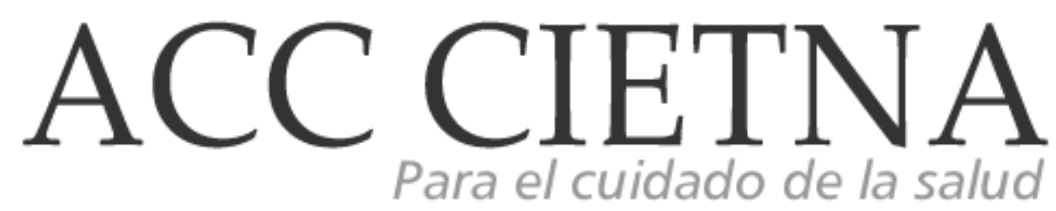

https://doi.org/10.35383/cietna.v6i2.251

\title{
Enfermedades ocupacionales del personal de enfermería y su relación con las condiciones de trabajo. Hospital Provincial Docente Belén de Lambayeque. 2018
}

\author{
Ojeda Mino, Miguel Angel ${ }^{1}$, Lázaro Alcántara, Elaine ${ }^{2}$
}

INFORMACIÓN DEL ARTÍCULO RESUMEN

\begin{tabular}{l} 
Historia del artículo: \\
Recibido el 06 de septiembre de 2019 \\
Aceptado el 06 de noviembre de 2019 \\
\hline Palabras clave: \\
Enfermedades Ocupacionales \\
Condiciones de trabajo \\
Personal de Enfermería
\end{tabular}

Este estudio tuvo por objetivo de determinar la relación que existe entre las enfermedades ocupacionales del personal de Enfermería y las condiciones de trabajo en el Hospital Provincial Docente Belén de Lambayeque. El tipo de investigación fue cuantitativo de correlación corte transversal. La muestra estuvo constituida por 127 trabajadores de enfermería, de nivel profesional y técnico, del Hospital Provincial Docente Belén Lambayeque. Se empleó, el cuestionario diseñado y validado por Blanch JM, Sahagún M y Cervantes G (2010), evaluándose las condiciones de trabajo: el factor organización y método, conformado por la escala de organización y método: regulación, y la escala organización y método: desarrollo y el factor organización y entorno que está conformado por la escala de organización y entorno material y la escala organización y entorno social. Entre las enfermedades ocupacionales y las condiciones de trabajo, no existe una relación directa, obteniéndose una $X^{2}=1.235 ; \mathrm{gl}=2$ y $\mathrm{p}=0.539$. Así mismo, se determinó que las enfermedades más frecuentes son: la lumbalgia, gastritis y síndrome de hombro doloroso, por otro lado, en las condiciones de trabajo en la escala de organización y entorno material lo califican como malo en un $48.8 \%$. Finalmente se determinó que no existe una relación significativa entre las enfermedades ocupacionales y las condiciones de trabajo.

\footnotetext{
'Licenciado en Enfermería en Hospital Docente Belén, Lambayeque, Perú. Email: miquelojedamino@gmail.com 2Doctora en Enfermería. Docente de pregrado y postgrado Universidad Católica Santo Toribio de Mogrovejo. Enfermera Asistencial Hospital Regional Policial, Chiclayo. Coordinadora del Grupo de Investigación en Enfermería, Trabajo, Historia y Salud. ORCID: https://orcid.org/0000-0002-7705-626X
} 
Occupational diseases of nursing staff and their relationship with working conditions. Provincial hospital Docente Belén de lambayeque. 2018

\section{ABSTRACT}

Keywords:

Occupational Diseases

Working Conditions

Nursing Staff
The purpose of this study was to determine the relationship that exists between the occupational diseases of nursing staff and the working conditions at Belén de Lambayeque Provincial Teaching Hospital. The type of research was quantitative cross-section correlation. The sample consisted of 127 nursing workers, professional and technical level, Belén Lambayeque Provincial Teaching Hospital. The questionnaire designed and validated by Blanch JM, Sahagún M and Cervantes $G$ (2010) was used, evaluating the working conditions: the organization and method factor, formed by the scale of organization and method: regulation, and the scale organization and method : development and the factor organization and environment that is conformed by the scale of organization and material environment and the scale of organization and social environment. Among occupational diseases and working conditions, there is no direct relationship, obtaining an $\mathrm{X} 2=1.235$; gl $=2$ and $p=0.539$. Likewise, it was determined that the most frequent diseases are: low back pain, gastritis and painful shoulder syndrome, on the other hand in the working conditions in the scale of organization and material environment they qualify it as bad in $48.8 \%$. Finally, it was determined that there is no direct relationship between occupational diseases and working conditions.

\section{Introducción}

La salud ocupacional es el conjunto de actividades que promueven el confort de los trabajadores, por lo que se previenen enfermedades relacionada con la actividad laboral. El personal de enfermería son parte fundamental del sector salud, de allí la importancia de las condiciones laborales en que ejercen su profesión.'En el 2017, la Organización Internacional del Trabajo (OIT) estima que las enfermedades ocupacionales causan más de 2.3 millones de muertes anuales, de los cuales, más de 300.000 mil son accidentes con lesiones ${ }^{2}$. La Organización Panamericana de la Salud (OPS) estima que en américa latina solo notifican enfermedades ocupacionales del $1 \%$ al $5 \%$, ya que sólo se registran casos que causan incapacidad sujeta a indemnización o bien éstas no son registradas como tales, sino que son clasificadas como enfermedades comunes 0 accidentes laborales ${ }^{3}$.

Por otra parte, el contexto laboral se evidencia problemas relacionadas con condiciones de trabajo, como el recargo de trabajo, jornadas prolongadas, turnos de noche, cambios de servicios. Además, un estudio realizado en Chile considera que las formas de trabajo afectan en la salud del personal de salud, y por consecuencia la calidad de la atención y la recuperación de los pacientes ${ }^{4}$. La saturación en el trabajo que se somete el personal de enfermería durante su jornada laboral, produce dolores lumbares $y$ agotamiento muscular, los cuales pueden padecer el personal de enfermería. En España la 
enfermedad más recurrente de los profesionales de enfermería son las enfermedades lumbares $(42,2 \%)$. Asimismo, en Estados Unidos la lumbalgia es causa de ausentismo en el personal de salud, con el $26,0 \%$ respectivamente ${ }^{5}$.

La labor del personal de salud es diferente a las otras profesiones por la atención que se brindan, de alta resonancia social y por poseer recursos humanos competentes. El personal de enfermería al estar propenso a enfermedades ocupacionales, presenta deterioro en la salud física como enfermedades musculares, gastrointestinales, sentimientos de impotencia, decepción, tristeza, insomnio ${ }^{6}$.

En el Perú, existe la ley $N^{a} 29783$, ley de Seguridad y Salud en el Trabajo, que protege a los trabajadores que presentan alguna enfermedad ocupacional y se encarga de establecer reglamentos de prevención en las empresas. Sin embargo, esta normativa no contempla de manera expresa indicadores para trabajadores en el sector salud. Esta misma normativa indica que las organizaciones sindicales, son las encargadas de la promoción, difusión y cumplimiento de la normativa. En este sentido, la norma establecería que en los hospitales se contará con un registro de enfermedades ocupacionales, sin embargo, el investigador al intentar recopilar información respecto a la casuística que precede a este tema, pudo determinar que no se cuenta con un registro fidedigno y menos registros que evidencien el monitoreo subsecuente de los resultados obtenidos en los exámenes físicos anuales que se le practican al personal de enfermería?.

El investigador a lo largo de su trayectoria como técnico de enfermería ha podido observar que, en el Hospital Provincial Docente Belén de Lambayeque, la situación de trabajo del personal de Enfermería, no cuenta con las mejores condiciones de trabajo presentando, condiciones inseguras, cabe destacar que esta realidad se repite en muchos hospitales del país, debido a la ausencia de política seguridad y salud ocupacional por lo tanto esto condiciona un ambiente hacinado, ocasionado principalmente por la antigua infraestructura que no permite una pronta mejora. Asimismo, el personal de enfermería realiza guardias diurnas y nocturnas dis minuyendo así las horas de sueño, exposición a radiaciones emitidas por los equipos tecnológicos, lo que repercute en la productividad y calidad de atención al paciente8.

Según las estadísticas del año 2014 al 2017, obtenidas en la sede de aplicación de este estudio, las enfermedades ocupacionales más comunes fueron la lumbalgia, dermatitis, gastritis, artrosis, migraña, faringoamigdalitis, conjuntivitis, infecciones urinarias, tendinitis y el síndrome de hombro doloroso. En el último año se ha podido determinar un promedio de sobrecarga laboral en el personal de enfermería.

En los centros hospitalarios se puede persuadir la carencia de política en salud ocupacional, ocasionando escaso personal idóneo en temas de seguridad y salud ocupacional, debido a que, poco presupuesto asigna el estado, observándose daños en la infraestructura como es el escenario de estudio que tiene más de 100 años de antigüedad, equipos en mal estado de conservación, mala limpieza y desinfección de los ambientes. las condiciones de trabajo y las bajas remuneraciones por servicios de terceros obligan al personal de enfermería a buscar otros trabajos en sus días de descanso post guardia, por lo que se condiciona que el personal de enfermería quiera fraccionar sus vacaciones en forma quincenal para evitar dejar de percibir el pago extraordinario de guardias al tomar vacaciones durante un mes por lo tanto tiene menos oportunidades de capacitación organización de los servicios y las relaciones interpersonales ${ }^{9}$.

Ante esta situación surgen las siguientes interrogantes: ¿Cómo son las condiciones en las que labora el personal de enfermería? ¿Cómo 
afectan las condiciones laborales del personal de enfermería en las enfermedades ocupacionales? ¿Cuáles son las enfermedades ocupacionales más frecuentes en el personal de enfermería? ¿Practica el personal de enfermería algunas medidas preventivas para evitar enfermedades ocupacionales? ¿La institución de salud les ofrece apoyo cuando se presenta una enfermedad ocupacional?

Un ambiente de trabajo que no cuenta con las condiciones apropiadas tiene implicancias negativas en la salud del personal de enfermería debido a que se someten a jornadas agotadoras, sobrecarga en el número de pacientes, deterioro de la infraestructura, dificultando trabajar en condiciones óptimas. El laborar en condiciones inadecuadas, lleva al personal de enfermería a presentar diversas enfermedades ocupacionales ${ }^{10}$.

En tal sentido, las enfermedades más recurrentes del personal de enfermería que labora en el Hospital Provincial Docente "Belén" de Lambayeque fueron la lumbalgia (50\%), gastritis(27\%) síndrome del hombro doloroso(24\%) migraña(15\%) y faringoamigdalitis, infección urinaria( $10 \%$ ),artrosis, el $3 \%$ dermatitis, el 2\% (6) tendinitis $2 \%$ conjuntivitis $1 \%$ Por lo que, estos resultados obtenidos, coinciden con los encontrados por Daqui donde las primordiales enfermedades ocupacionales que comparece el personal de enfermería fueron el estrés laboral y lumbalgia con un $28 \%$, un $23 \%$ las varices, un $16 \%$ alergias y las ITU, un $11 \%$ cefalea, un $8 \%$ gastritis, el $7 \%$ tendinitis y dermatitis, y un $4 \%$ cervicalgia en la cual se pudo comprobar en una de las escalas de organización y entorno material las malas condiciones en que se desenvuelve el personal de Enfermería y cómo ésta impacto en su salud. Por lo que estos resultados de este estudio, servirán como antecedente para futuras investigaciones que pretendan mejorar las condiciones de trabajo, ya que permiten conocer y profundizar la realidad de las condiciones en la que labora el personal de enfermería ${ }^{11}$.

La labor del personal de Enfermería demanda esfuerzo físico, lo que, asociado a posturas corporales inapropiadas, deterioro de la infraestructura, jornadas agotadoras, sobrecarga en el número de pacientes y a las malas condiciones de trabajo ocasiona repercusiones negativas en su salud ${ }^{12}$.

\section{Metodología}

La investigación fue cuantitativa porque se determinó la relación que existe entre las enfermedades ocupacionales y condiciones de trabajo del personal de Enfermería, lo que conllevó a probar las hipótesis formuladas, mediante pruebas estadísticas, estableciendo el comportamiento de las variables. El alcance del estudio fue descriptivo, porque se permitió especificar las propiedades y características de las variables mostrando con precisión correlacionar entre las mismas. La investigación fue con diseño, correlacional, transversal. No experimental, se buscó establecer relación directa de causa y efecto entre las variables. Se pretendió examinar si los cambios de la variable dependiente (enfermedad ocupacional) están relacionados con los cambios de la variable independiente (condiciones de trabajo), se describió la relación lineal entre estas dos variables evaluando el grado de asociación entre las mismas sin llegar a determinar la casualidad, con diseño transaccional: porque se describió la relación entre las variables en un momento determinado de tiempo ${ }^{13}$.

La información fue recolectada en los servicios de emergencia, sala de operaciones, ginecología, neonatología cirugía, traumatología y medicina, pediatría en diferentes días al total de la población. Esta población estuvo constituida por 127 trabajadores de enfermería, constituyendo el total de la población. 54 profesionales de enfermería y 73 técnicos de enfermería. Esta 
población estuvo constituida por Personal de enfermería: Profesionales de enfermería y técnicos de enfermería del hospital en estudio. Sexo: Hombres y mujeres, condición laboral. Nombrados y contratados (CAS).

La determinación de la muestra fue no probabilística. Con la finalidad de obtener mayor confiabilidad en los resultados se trabajó con la población como universo muestral, pero segmentados por grupos ocupacional: profesionales de enfermería y técnicos de enfermería. La técnica que se utilizó, fue la encuesta y el instrumento, un cuestionario que constó de dos partes. Los datos sociodemográficos, datos laborales y las condiciones de trabajo que consta de dos factores. El instrumento que se utilizó fue diseñado y validado por Blanch JM, Sahagún $M$ y Cervantes $G$ en el año 2010, constituidos por escalas que tienen un formato de respuesta de elección múltiple con opciones que van de 0 (significa valor pésimo) a 10 (significa valor óptimo). El cuestionario se aplicó con consentimiento informado. El análisis de fiabilidad realizado por Blanch et al obtuvo un valor global de (58) a alfa de Crombach de 0,96 en el c-CT oscilando los valores entre un mínimo de 0,83 en la escala de entorno social y un máximo de 0,97 en la escala de adaptación persona-organización. En la muestra de estudio, los valores de alfa de Crombach obtenidos fueron similares en términos de consistencia interna a los obtenidos por Blanch et, a los valores obtenidos en la muestra de investigación ${ }^{14}$.

La presente investigación fue aprobada por el comité de ética, siendo ratificada mediante resolución de Consejo de Facultad N²476-2018USAT-FMED.Luego se procedió a realizar los trámites administrativos para la ejecución de la investigación y se obtuvo la autorización respectiva del Hospital Provincial Docente Belén Lambayeque con Reg. N 40- PL-2018. Luego se aplicó el instrumento en una prueba piloto en otro escenario de estudio a 25 voluntarios entre profesional de Enfermería y Técnicos de Enfermería comprobándose que las preguntas establecidas en la guía de entrevista fueron entendidas y las respuestas respondieron al objeto de estudio.

Posteriormente se procedió a recolectar los datos de dicho cuestionario en los diferentes servicios del Hospital Belén en diferentes días, iniciándose a partir del mes de agosto aplicándose al personal de Enfermería, previamente se explico acerca del objetivo de la investigación y finalmente el consentimiento informado, basado según los criterios de inclusión y exclusión establecidos.

Después de haber obtenido la información, llenado de cuestionarios y procediendo a la calificación, los datos han sido organizados, tabulados y graficados mediante el programa Excel, para ser transferida posteriormente al programa (SPSS versión 23). Una vez transferida los datos al programa estadístico mencionado anteriormente, se procedió a realizar la validación del instrumento utilizando para ello la prueba de confiabilidad del Alfa de Cron Bach para evaluar la consistencia interna de los ítems de cada una de las 127 encuestas utilizadas.

Luego se calculó la prueba de normalidad de kolmogorov $(n>30)$ para el puntaje total de las condiciones de trabajo, con el objetivo de averiguar si las valoraciones de los 127 encuestados siguen una distribución normal, cuyo resultado fue positivo. Antes de realizar el análisis para cumplir con cada objetivo específicos planteados, así como el objetivo general, se realizó una tabla bivariada de los factores sociodemográficos con el fin de poder tener una visión más amplia de nuestra población.

Con respecto al objetivo general se utilizó una tabla de doble entrada o de contingencia con sus respectivos porcentajes, y para poder responder a la hipótesis planteada se calculó el 
coeficiente de correlación de Tau b Kendall con el fin de saber el grado de asociación entre estas dos variables. Para el primer objetivo específico se utilizó una tabla unidimensional evaluadas al 100\% por cada fila, dado a que cada encuestados puede tener más de 2 enfermedades ocupacionales.

Respecto al segundo y último objetivo específico, se utilizó para cada una de las escalas tablas unidimensionales con sus respectivos porcentajes y una tabla unidimensional general sobre condiciones de trabajo con el fin de conocer cuales es el nivel de valoración con respecto a dicha variable. Cabe mencionar, que se utilizaron de manera adicional, tablas unidimensionales correspondientes a las correlaciones de Pearson, para saber qué tan correlacionadas están entre las escalas de las condiciones de trabajo.

Dado a que las puntuaciones de las condiciones de trabajo siguen una distribución normal se aplicaron estadísticos paramétricos, análisis de varianza, prueba $t$ de student para diferencias de medias, con el fin de conocer si las variables de Enfermedades ocupacionales y Condiciones de trabajo de manera global, son estadísticamente significativas, siempre y cuando el $p$-valor de la tabla es menor que 0.05 .

Teniendo en cuenta el modelo personalista ontológico de Elio Sgreccia, los éticos que rigieron la presente investigación fueron: El valor fundamental de la vida: Al atribuir este principio, se consideró la vida como un bien primordial, valorando su dignidad de la persona, encaminando al personal de Enfermería, como un ser integro, único, racional, inteligente con múltiples dimensiones, con valores y principios, y el respeto de su autonomía procurando en todo momento proteger su identidad, tomando en cuenta su independencia y su consentimiento en el estudio. El principio de totalidad o principio terapéutico: En esta investigación el personal de Enfermería del Hospital Belén Lambayeque, fue respetados en su totalidad, integridad y valor como persona, por lo que, se les informo sobre su participación en esta investigación. Este principio refuerza el valor de la vida humana, pero requiere de condiciones para aplicarse ${ }^{15}$.

\section{Resultados, análisis y discusión}

Tabla $N^{\circ} 1$

Factores Sociodemográficos del personal de Enfermería del Hospital Provincial Docente Belén Lambayeque 2018

\begin{tabular}{|c|c|c|c|c|c|c|c|}
\hline \multirow{2}{*}{\multicolumn{2}{|c|}{$\begin{array}{c}\text { Datos Sociodemográficos y } \\
\text { laborales }\end{array}$}} & \multicolumn{6}{|c|}{ Según Edad } \\
\hline & & \multicolumn{2}{|c|}{$\begin{array}{l}25 \text { a } 35 \\
\text { años }\end{array}$} & \multicolumn{2}{|c|}{$\begin{array}{l}36 \text { a } 46 \\
\text { años }\end{array}$} & \multicolumn{2}{|c|}{$\begin{array}{l}\text { más de } \\
47 \text { años }\end{array}$} \\
\hline & & $\mathrm{n}$ & $\%$ & $\mathrm{n}$ & $\%$ & $\mathrm{n}$ & $\%$ \\
\hline \multirow{2}{*}{ Sexo } & Femenino & 18 & 15.5 & 30 & 25.9 & 68 & 58.6 \\
\hline & Masculino & 2 & 18.2 & 3 & 27.3 & 6 & 54.5 \\
\hline \multirow{8}{*}{$\begin{array}{l}\text { Servicios } \\
\text { Laborales }\end{array}$} & Medicina & 4 & 21.1 & 7 & 36.8 & 8 & 42.1 \\
\hline & Emergencia & 5 & 17.2 & 7 & 24.1 & 17 & 58.6 \\
\hline & Pediatría & 2 & 18.2 & 2 & 18.2 & 7 & 63.6 \\
\hline & Neonatología & 2 & 13.3 & 3 & 20 & 10 & 66.7 \\
\hline & $\begin{array}{c}\text { Sala de } \\
\text { Operaciones }\end{array}$ & 4 & 18.2 & 5 & 22.7 & 13 & 59.1 \\
\hline & Cirugía & 0 & 0 & 4 & 50 & 4 & 50 \\
\hline & $\begin{array}{l}\text { Traumatología } \\
\text { y especializado }\end{array}$ & 1 & 11.1 & 3 & 33.3 & 5 & 55.6 \\
\hline & Ginecología & 2 & 18.2 & 2 & 18.2 & 7 & 63.6 \\
\hline \multirow{4}{*}{$\begin{array}{l}\text { Tiempo de } \\
\text { labor en la } \\
\text { institución }\end{array}$} & 1 año a 5 años & 12 & 48 & 9 & 36 & 4 & 16 \\
\hline & $\begin{array}{c}6 \text { años a } 10 \\
\text { años }\end{array}$ & 7 & 35 & 8 & 40 & 5 & 25 \\
\hline & $\begin{array}{l}11 \text { años a } 20 \\
\text { años }\end{array}$ & 1 & 4 & 13 & 52 & 11 & 44 \\
\hline & $\begin{array}{l}\text { Más de } 20 \\
\text { años }\end{array}$ & 0 & 0 & 3 & 5.3 & 54 & 94.7 \\
\hline \multirow{2}{*}{$\begin{array}{l}\text { Situación } \\
\text { Laboral }\end{array}$} & Nombrado & 9 & 8.5 & 27 & 25.5 & 70 & 66 \\
\hline & Contratado & 11 & 52.4 & 6 & 28.6 & 4 & 19 \\
\hline \multirow{2}{*}{$\begin{array}{c}\text { Grupo } \\
\text { ocupacional }\end{array}$} & $\begin{array}{c}\text { Profesional de } \\
\text { enfermería }\end{array}$ & 10 & 18.5 & 9 & 16.7 & 35 & 64.8 \\
\hline & $\begin{array}{l}\text { Técnico en } \\
\text { enfermería }\end{array}$ & 10 & 13.9 & 25 & 33.3 & 38 & 52.8 \\
\hline \multirow{4}{*}{$\begin{array}{l}\text { Turno de } \\
\text { trabajo } \\
\text { habitual }\end{array}$} & Mañana & 4 & 28.6 & 2 & 14.3 & 8 & 57.1 \\
\hline & Solo tarde & 0 & 0 & 1 & 100 & 0 & 0 \\
\hline & $\begin{array}{c}\text { Diurna de } 12 \\
\text { horas }\end{array}$ & 16 & 14.3 & 30 & 26.8 & 66 & 58.9 \\
\hline & $\begin{array}{l}\text { Nocturna de } \\
12 \text { horas }\end{array}$ & 16 & 14.3 & 30 & 26.8 & 66 & 58.9 \\
\hline $\begin{array}{c}\text { En los } \\
\text { últimos } 12 \\
\text { meses }\end{array}$ & $\begin{array}{c}\text { No he estado } \\
\text { en licencia por } \\
\text { enfermedad }\end{array}$ & 19 & 18.1 & 28 & 26.7 & 58 & 55.2 \\
\hline
\end{tabular}


$\begin{array}{llllllll}\text { ¿Cuánto } \quad \text { Menor a } 1 \text { mes } & 1 & 5.3 & 5 & 26.3 & 13 & 68.4\end{array}$ tiempo ha estado de licencia por De 1 a 3 meses

enfermería?

En los

últimos 3

Programadas

$19 \quad 15.3 \quad 33$

meses ¿Cuál

ha sido el

promedio

de horas de

Extras

100

trabajo?

Fuente: Encuesta aplicada al personal de enfermería del Hospital Provincial Docente Belén. Lambayeque. 2018

En relación con los diferentes factores sociodemográficos, entre los cuales tenemos que respecto al sexo, relacionado con la edad, del $100 \%$ (127), el 58.6\% (68) son mayores 47 años, y el 15.5\% (18) tienen entre 25 a 35 años son del sexo femenino; mientras que el $54.5 \%$ (6) tienen más de 47 años, y el 18.2\% (2) tienen entre 25 y 35 años, del sexo masculino, por lo que se evidencia que la mayoría del personal de enfermería, tienen más de 47 años y son de sexo femenino. Con respecto a los servicios laborales y su relación con la edad se tiene que, del 100\% (127), el 63\% (7) que labora en el servicio de ginecología, tiene más de 47 años, y el $21 \%$ (4) del servicio de medicina tiene entre 25 a 35 años. En relación a la situación laboral, se tiene que del 100\% (127), el 66\% (70) tiene más de 47 años, y son nombrados. Asimismo, el 52.4\% (11) tiene entre 25 a 35 años y son contratados. Por otro lado, según el grupo ocupacional, el $64.8 \%$ (35) que tienen más de 47 años de edad y el 18.5\% (10) que tienen entre 25 a 35 años de edad son profesionales de enfermería; no obstante, el $52.8 \%$ (38) que tienen más de 47 años de edad, y el 13.9\% (10) que tienen entre 25 a 35 años de edad son técnicos en enfermería. Según el tiempo de trabajo el 94.7\% (54) tiene más de 20 años laborando en la institución, y el 16\% (4) tiene de 1 a 5 años, teniendo todos ellos más de 47 años de edad. En relación al turno de trabajo habitual las guardias de 12 horas tanto diurnas como nocturnas del 100\% (127), el 58.9\% (66) tienen más de 47 años de edad y los turnos de 6 horas, el $28.6 \%$ tienen entre 25 y 35 años. Por tal motivo esta investigación tiene relación con el estudio realizado por Ramirez ${ }^{16}$ donde se encontró la disimilitud en la edad de las personas, ya que las mayores secuelas para la salud vinculados con la turnicidad laboral, ocurren con el incremento de la edad.

Respecto al tiempo de licencia en los últimos 12 meses, del 100\% (127), el 100\% (3) manifestaron haber tenido licencia de 1 a 3 meses $y$ el $68.4 \%$ (13) tuvo una licencia menor a un mes. Lo que coincide con el estudio realizado por Díaz ${ }^{17}$ donde menciona que, de todo el personal de enfermería, el 38.46\% tienen entre 47 a más años, y el $18.46 \%$ entre 25 a 35 años, siendo el $9.23 \%$ de sexo masculino y $90.77 \%$ de sexo femenino.

Así pues, podemos observar que la mayoría representa al sexo femenino, y debido a sus características físicas y al trabajo que realiza durante su jornada laboral, tienden a ser más vulnerables por lo que están más propensas a adquirir diferentes enfermedades ocupacionales, por lo que estos resultados están en concordancia con lo publicado por el INEI, que según el censo del 2017 la población femenina asciende a $50.8 \%$ mientras que la masculina es de $49.2 \%^{18}$.

\section{Tabla No 2}

Relación que existe entre las enfermedades ocupacionales del personal de enfermería y las condiciones de trabajo en el Hospital Provincial Docente Belén de Lambayeque

\begin{tabular}{lcccc}
\hline \multirow{2}{*}{ Condiciones de Trabajo } & \multicolumn{3}{c}{ Nnfermedad Ocupacional } \\
\cline { 2 - 5 } & $\mathrm{N}$ & $\%$ & $\mathrm{n}$ & $\%$ \\
\cline { 2 - 5 } & 0 & 0 & 42 & 33.6 \\
Malo & 2 & 100 & 77 & 61.6 \\
Bueno & 0 & 0 & 6 & 4.8 \\
\hline Total & 2 & 100 & 125 & 100 \\
\hline \multicolumn{5}{c}{$\mathrm{X}^{2}=1.235 \mathrm{gl}=2 \mathrm{p}=0.539$} \\
\hline
\end{tabular}

Fuente: Encuesta aplicada al personal de enfermería de Hospital Provincial Docente Belén. Lambayeque. 2018 
En cuanto a la relación que existe entre las enfermedades ocupacionales y las condiciones de trabajo, del 100\% (127), el 61.6\% (77) presentaron enfermedades ocupacionales, clasificando como bueno la condición de trabajo, sin embargo, un pequeño porcentaje, representado por 33.6\% (42) clasificaron como malo la condición de trabajo.

De acuerdo a los estadísticos para prueba de hipótesis aplicados $\left(\mathrm{X}^{2}=1.235 \mathrm{gl}=2 \mathrm{p}=0.539\right)$ se acepta Hipótesis nula (H0) y se concluye que no existe relación significativa entre enfermedad ocupacional y condiciones de trabajo, con un nivel de significancia del 95\%. Queda demostrado que, las condiciones de trabajo, no están directamente relacionadas al potencial de enfermedad del trabajador, ya que las condiciones de trabajo son definidas por un conjunto de factores que incluyen horas trabajadas, remuneración salarial, organización laboral, conocimiento en relación a la misma, higiene y seguridad en el trabajo, ergonomía, disponibilidad de servicios sociales para los trabajadores, relaciones laborales, individualidad de los trabajadores cada trabajador, el estatus socioeconómico y el país la política, entre otros 19 .

Tabla $N^{\circ} 3$

Enfermedades ocupacionales recurrentes en el personal de enfermería del Hospital Provincial Docente Belén. Lambayeque 2018.

\begin{tabular}{llllllll}
\hline \multirow{2}{*}{$\begin{array}{l}\text { Onfermedades } \\
\text { Ocupacionales }\end{array}$} & \multicolumn{3}{c}{ Sí } & \multicolumn{3}{c}{ No } & \multirow{2}{*}{ Total } \\
\cline { 2 - 5 } & $\mathrm{n}$ & $\%$ & $\mathrm{n}$ & $\%$ & & \\
\hline \hline Lumbalgia & 63 & 50 & 64 & 50 & 127 & 100 \\
Dermatitis & 4 & 3 & 123 & 97 & 127 & 100 \\
Gastritis & 34 & 27 & 93 & 73 & 127 & 100 \\
Artrosis & 10 & 8 & 117 & 92 & 127 & 100 \\
Faringoamigdalitis & 19 & 15 & 108 & 85 & 127 & 100 \\
Conjuntivitis & 1 & 1 & 126 & 99 & 127 & 100 \\
Infección Urinaria & 13 & 10 & 114 & 87 & 127 & 100 \\
Tendinitis & 6 & 2 & 121 & 95 & 127 & 100 \\
Migraña & 19 & 15 & 108 & 85 & 127 & 100 \\
Síndrome Hombro & 30 & 24 & 97 & 76 & 127 & 100 \\
Doloroso & & & & & 76 & & \\
\hline
\end{tabular}

Fuente: Encuesta aplicada al personal de enfermería del Hospital Provincial Docente Belén. Lambayeque. 2018
En esta investigación, se encontró que del $100 \%$ (127), el 50\% (63) presentan lumbalgias, el $27 \%$ (34) gastritis, el 24\% (30) síndrome del hombro doloroso, el $15 \%$ (19) migraña y faringoamigdalitis, el 10\% (13) infección urinaria, el $8 \%(10)$ artrosis, el 3\% (4) dermatitis, el 2\% (6) tendinitis y el 1\% (1) conjuntivitis. Por lo que, estos resultados obtenidos, coinciden con los encontrados por Daqui donde las primordiales enfermedades ocupacionales que comparece el personal de enfermería fueron el estrés laboral y lumbalgia con un $28 \%$, un $23 \%$ las varices, un $16 \%$ alergias y las ITU, un $11 \%$ cefalea, un $8 \%$ gastritis, el $7 \%$ tendinitis y dermatitis, y un $4 \%$ cervicalgia 20 .

Tabla $\mathbf{N}^{\circ} 4$

Condiciones de trabajo del personal de enfermería del Hospital Provincial Docente Belén. Lambayeque 2018.

\begin{tabular}{|c|c|c|}
\hline $\begin{array}{l}\text { Condiciones } \\
\text { trabajo }\end{array}$ & $\mathrm{N}$ & $\%$ \\
\hline Malo & 42 & 33.1 \\
\hline Bueno & 79 & 62.2 \\
\hline Optimo & 6 & 4.7 \\
\hline Total & 127 & 100.0 \\
\hline
\end{tabular}

Fuente: Encuesta aplicada al personal de enfermería del Hospital Provincial Docente Belén. Lambayeque. 2018

Dentro de las condiciones de trabajo el $62.2 \%$ del personal de enfermería lo califica como bueno y el $33.1 \%$ lo califica como malo. Sin embargo, en un estudio realizado por Borges. Demostró la precariedad de las condiciones de trabajo en que labora el personal de enfermería ${ }^{21}$. Así también, en otro estudio realizado por Merchán, manifiesta que los profesionales enfermeros en Chile se desenvuelven en las condiciones laborales marcadas por la carencia de materiales, alta demanda laboral y limitados recursos. Por lo que la precariedad de las condiciones de trabajo, acarrean perjuicios en la vida cotidiana privada de este trabajador ${ }^{22}$.

Las condiciones de trabajo del personal de enfermería, han experimentado profundos cambios generales en el mundo del trabajo donde 
se ha analizado el impacto de las condiciones de trabajo en el bienestar y el desempeño profesional, así como sus múltiples efectos secundarios en la salud ocupacional y la calidad del servicio prestado. Las condiciones laborales del personal de enfermería establecen un riesgo para su salud física y mental, por cuanto la práctica de cuidar acarrea una sobrecarga laboral y emocional, debido a los diversos dilemas que proceden de la atención y de los sistemas de salud. Por lo que las enfermeras ven apercibidas su complacencia laboral y profesional ${ }^{23}$.

\section{Tabla $\mathbf{N}^{\circ} 5$}

Organización y entorno material del personal de enfermería del Hospital Provincial Docente Belén.

Lambayeque 2018.

\begin{tabular}{lcc}
\hline Entorno Material & $\mathrm{N}$ & $\%$ \\
\hline \hline Pésimo & 6 & 4.7 \\
Malo & 62 & 48.8 \\
Bueno & 52 & 40.9 \\
Optimo & 7 & 5.5 \\
\hline Total & 127 & 100.0 \\
\hline
\end{tabular}

Fuente: Encuesta aplicada al personal de enfermería del Hospital Provincial Docente Belén. Lambayeque. 2018

Del personal de enfermería encuestada, el $49 \%$ califican como malo la organización y entorno material, mientras que $41 \%$ lo califica como bueno. Por lo que coincide con un estudio realizado en Colombia por Leguizamón I, Gómez V. en lo referente a las condiciones de trabajo y retribución de materiales de trabajo, valora que las enfermeras se confrontan a obstáculos como ambiente reducido, temperatura inapropiada, equipos y materiales incompletos e inseguros 24 .

En Barcelona en una investigación que, en la escala de organización, entorno material y prevención de riesgos, las enfermeras expresaron que el entorno físico requiere de un acondicionamiento de las instalaciones, los equipos y los recursos materiales. Asimismo, reconocieron situaciones de riesgo laboral como la sobrecarga, presión laboral, el estrés, el cansancio, la despersonalización, la desmotivación, la insatisfacción y los abusos de poder, se evidencio la deficiencia en la asignación de equipos y recursos materiales indispensables para realizar su labor. Por tanto, el artículo 9, numeral D, de la ley que rige el ejercicio profesional de enfermería en Perú establece como un derecho contar con los recursos materiales y equipos necesarios $y$ adecuados para cumplir sus funciones de manera infalible y seguro, que le permitan brindar servicios de calidad 25 .

\section{Conclusiones}

De manera global, no existe relación significativa entre las Enfermedades ocupacionales y las condiciones de trabajo, según las escalas aplicadas. Esto podría explicarse a partir de la calificaron de "buena" las condiciones de trabajo en las que laboran las enfermeras y la variación evaluativa que ha tenido la escala en sus diferentes dimensiones.

Las enfermedades ocupacionales más recurrentes: la lumbalgia con el $50 \%$, gastritis el $27 \%$, síndrome del hombro doloroso el $24 \%$. Y un $1 \%$ con conjuntivitis, resultados que se encuentran dentro de las tendencias nacionales e internacionales con las que se ha contrastado.

Las condiciones de trabajo, evaluado en dos factores y cuatro escalas, demuestran que los factores: Organización y método Regulación y Desarrollo; y Organización y Entorno social, calificaron como buena. En cambio, en la escala Organización y entorno material la calificaron como malo, debido a que existe precariedad en el entorno físico, instalaciones y equipamiento, recursos materiales, prevención de riesgos laborales, limpieza y seguridad, que propician condiciones de trabajo inapropiadas. 


\section{Bibliografía}

1. Rodríguez C, Bravo C. formulario para la descripción del trabajo de grado. [cited 2018 25]; available from: http://www.javeriana.edu.co/biblos/tesis/enf ermeria/20092/definitiva/tesis21.pdf

2. Organización Internacional Del Trabajo. urge a una acción mundial para combatir las enfermedades profesionales. [cited $2018 \mathrm{apr}$ 8]; available from: http://www.ilo.org/global/about-theilo/newsroom/news/wcms_21 1645/lang-es/index.htm

3. Organización Mundial de la Salud. Enfermedades Profesionales en las Américas [internet]. paho.org. [cited 2018 apr 8]. p. 1. available

from: http://www.paho.org/per/index.php?option = com_content\&view $=$ article\&id=2114:opsoms-estima-que-hay-770-nuevos-casosdiarios-personas-enfermedadesprofesionales-americas\&itemid $=900$

4. Canales M, Valenzuela S. Condiciones de Trabajo de los profesionales de Enfermería en Chile. [internet]. 2016;13(3):178-86. available from:

http://linkinghub.elsevier.com/retrieve/pii/s 1665706316300215

5. Rivera M, Sanmiguel M, Serrano L, Nava M, et al. Factores Asociados a Lesiones MúsculoEsqueléticas por Carga en Trabajadores Hospitalarios de la ciudad de torreón, Coahuila, México. [internet]. 2015 aug [cited 2018 apr 5];17(53):144-9. available from: http://www.scielo.cl/scielo.php?script=sci_ar ttext\&pid $=$ s0718 $24492015000200008 \& \operatorname{lng}=e n \& n r m=$ iso $\&$ t $\ln$ $\mathrm{g}=\mathrm{en}$

6. Universidad nacional de Santiago del estero. Facultad de Humanidades CS y de la S. trabajo y sociedad. Trab y Soc [internet]. 2017 [cited 2018 apr 5]; (28):11-35. available from: http://www.scielo.org.ar/scielo.php?script=s ci_arttext\&pid=s1 514-68712017000100002
7. Ley de seguridad y salud en el trabajo del Perú [internet]. 2011 [cited 2018 apr 7]; 29783:113. Available from: http://www.munlima.gob.pe/images/descarg as/seguridad-salud-en-el-trabajo/ley 29783 _ ley de seguridad y salud en el trabajo.pdf

8. Plan nacional gestión seguridad y salud ocupacional en hospitales del Minsa, 2018 gestión de la seguridad y salud [cited 2018 jun 15]; available from: http://www.digesa.minsa.gob.pe/dso/inform es/propuesta técnica del plan nacional de gestión en seguridad y salud ocupacional en hospitales.

9. Mauro M, Pinheiro M, Silva G. condições de trabalho da enfermagem nas enfermarias de um hospital universitário. Esc anna nery [internet]. 2010 jun [cited 2018 Apr. 18]; 14(2):244-52. Available from: http://www.scielo.br/scielo.php?script=sci_a rttext\&pid =s 1414

$81452010000200006 \& \operatorname{lng}=p t \& \operatorname{lng}=p t$

10. Canales M. condiciones de trabajo de los profesionales de enfermería en chile. Enfermería univ [internet]. 2016 jul [cited 2018 Apr. 6];13(3):178-86. available from: http://linkinghub.elsevier.com/retrieve/pii/s 1665706316300215

11. Hernández K. enfermedades más frecuentes en el personal de enfermería de las unidades de salud del primer nivel del área 2 de pastaza en el año 2013-2014. 2014 [cited 2018 apr 28]; available from: http://dspace.unl.edu.ec/handle/ 123456789 $/ 12167$

12. Següel F, Valenzuela $S$, Sanhueza O. el trabajo del profesional de enfermería: revisión de la literatura. Cienc y enfermería [internet]. 2015 Aug. [cited 2018 apr 5];21 (2):1 1-20. available from:

http://www.scielo.cl/scielo.php?script=sci_ar ttext\&pid=s0717-

$95532015000200002 \& \operatorname{lng}=$ en\&nrm $=$ iso\&t $\ln$ $\mathrm{g}=\mathrm{en}$ 
13. Hernández R. Metodología de la Investigación 6ta ed. Mc Graw Hill

14. Lázaro A. Calidad de vida laboral de las enfermeras evaluación y propuestas de mejora. [cited 2018 may 17]; available from: https://www.tdx.cat/bitstream/handle/ 1080 3/404610/agl_tesis.pdf?sequence $=1$

15. Fundamentos y Biomédica é. manual de bioética. [cited 2018 may 20]; available from: http://www2.congreso.gob.pe/sicr/cendocbi b/con4_uibd.nsf/2863c5229d0ae0cb05257d 10005241b6/\$file/resena_13543_mar091__indice.pdf

16. Aular M. Factores asociados al ausentismo laboral del personal de enfermería en el área de quirófano de un hospital materno infantil ubicado en Guacara- estado Carabobo. 2016 [cited 2018 Nov 9]; Available from: http://riuc.bc.uc.edu.ve/handle/123456789/ 4277

17. Ramírez N, ParaviT, V. Riesgo de los turnos nocturnos en la salud integral del profesional de enfermería. Índex de Enfermería [Internet]. [cited 2018 Apr 5];22(3):152-5. Available from:

http://scielo.isciii.es/scielo.php?script=sci_ar ttext\&pid $=$ S1 132-

$12962013000200008 \& \operatorname{lng}=$ en\&nrm $=$ iso\&tln $\mathrm{g}=\mathrm{en}$

18. Instituto Nacional de Estadística e Informática INEI [Internet]. Tecnologías de la información y comunicación. 2017 [cited 2018 Nov 9]. Available from: https://www.inei.gob.pe/estadisticas/indicetematico/tecnologias-de-la-informacion-ytelecomunicaciones/

19. Universidade fedral de minas gerais. escola de enfermagem $s$, souza nvd de o, farias snp. reme. [Internet]. vol. 19, reme rev. min. enferm. 2015 [cited 2018 apr 18]. 43-48 p. available from: http://pesquisa.bvsalud.org/portal/resource /pt/lil-768466

20. León C, Daqui J, Enfermedades Ocupacionales del personal de enfermería y su relación con el ausentismo laboral en el hospital de
Riobamba. \376\3772010 [cited 2018 Apr 25]; Available from: http://dspace.unach.edu.ec/bitstream/5100 0/259/1/UNACH-EC- Enfer-201 1-0009.pdf

21. Borges $A$, condiciones de trabajo Alto Riesgo. Disponible

en: file:// C:/Users/miguel/Downloads/DialnetPersonalDeEnfermeria-464197\%20(1).pdf

22. Luan HD, Hai NT, Xanh PT, et al. Musculoskeletal Disorders: Prevalence and Associated Factors among District Hospital Nurses in Haiphong, Vietnam. Biomed Res Int [Internet]. 2018 Aug 26 [cited 2019 Mar 30]; 2018:1-9. Available from: http://www.ncbi.nlm.nih.gov/pubmed/3022 5248

23. Merchán $P$, Jurado $A$. nuevas condiciones laborales para el profesional de enfermería. [cited 2018 Apr 8]; Available from: http://www.bvsde.ops-

oms.org/foro_hispano/BVS/bvsacd/cd49/nue vas.pdf

24. Molero M Acercamiento Multidisciplinar a la salud.: $\quad$ ASUNIVEP 2017 https://www.formacionasunivep..com/Vciise/ files/libros /LIBRO_2.pdf\#page $=237$

25. Leguizamón L, Gómez V. Condiciones laborales y de salud en enfermeras de Santafé de Bogotá. Rev. International Journal of Clinical and Health Psychology file:// /C:/Users/miguel/Desktop/tesis\%20co ndicones\%20cololom.pdf 